\title{
Probing the trilinear Higgs boson self-coupling at the high-luminosity LHC via multivariate analysis
}

\author{
Jung Chang, ${ }^{1}$ Kingman Cheung, ${ }^{2,3,4}$ Jae Sik Lee®, ${ }^{1,5}$ and Jubin Park ${ }^{5,1}$ \\ ${ }^{1}$ Department of Physics, Chonnam National University, \\ 300 Yongbong-dong, Buk-gu, Gwangju 500-757, Republic of Korea \\ ${ }^{2}$ Physics Division, National Center for Theoretical Sciences, Hsinchu 300, Taiwan \\ ${ }^{3}$ Division of Quantum Phases and Devices, School of Physics, Konkuk University, \\ Seoul 143-701, Republic of Korea \\ ${ }^{4}$ Department of Physics, National Tsing Hua University, Hsinchu 300, Taiwan \\ ${ }^{5}$ Institute for Universe and Elementary Particles, Chonnam National University, \\ 300 Yongbong-dong, Buk-gu, Gwangju 500-757, Republic of Korea
}

(Received 13 August 2019; published 3 January 2020)

\begin{abstract}
We perform a multivariate analysis of Higgs-pair production in $H H \rightarrow b \bar{b} \gamma \gamma$ channel at the HL-LHC to probe the trilinear Higgs self-coupling (THSC) $\lambda_{3 H}$, which takes the value of 1 in the standard model (SM). We consider all known background processes. For the signal we are the first to adopt the most recent event generator of POWHEG-BOX-V2 to exploit the next-to-leading order (NLO) distributions for the toolkit for multivariate data analysis (TMVA), taking account of the full top-quark mass dependence. Through boosted decision tree (BDT) analysis trained for $\lambda_{3 H}=1$, we find that the significance can reach up to 1.95 with about 9 signal and 18 background events. In addition, the THSC can be constrained to $1.00<$ $\lambda_{3 H}<6.22$ at $95 \%$ confidence level (CL). We also perform a likelihood fitting of $M_{\gamma \gamma b b}$ distribution and find the $1 \sigma$ confidence interval (CI) of $0.1<\lambda_{3 H}<2.2 \cup 5.4<\lambda_{3 H}<6.6$ for the $\lambda_{3 H}=1$ nominal set. On the other hand, using BDTs trained for each value of $\lambda_{3 H}$, we find a bulk region of $0.5 \lesssim \lambda_{3 H} \lesssim 4.5$, for which it is hard to pin down the THSC.
\end{abstract}

DOI: 10.1103/PhysRevD.101.016004

\section{INTRODUCTION}

Since the discovery of the Higgs boson in 2012 [1], the most pressing question is to understand the underlying mechanism for electroweak symmetry breaking (EWSB). There is no particular reason why the EWSB sector only consists of a single Higgs doublet. Indeed, the simplest version suffers from the so-called gauge hierarchy problem. After completing Run I and II at the LHC, the identity of the Higgs boson has been established. It is best described as the standard model (SM) Higgs boson [2], although there is an upward trend in the overall signal strength [3]. All current measurements of the Higgs boson properties confine to the couplings of the Higgs boson to the SM particles, like gauge bosons and fermions. However, the self-couplings of the Higgs boson are not established at all, which depends on the dynamics of the EWSB sector. The self-couplings of the Higgs boson can be very different

Published by the American Physical Society under the terms of the Creative Commons Attribution 4.0 International license. Further distribution of this work must maintain attribution to the author(s) and the published article's title, journal citation, and DOI. Funded by SCOAP ${ }^{3}$. between the SM and other extensions of the EWSB sector, like the two-Higgs doublet models (2HDM), and minimal supersymmetric extension of the standard model. Higgs boson pair production at the LHC provides a very useful avenue to investigate the THSC [4-6]. There is a vast literature on Higgs-pair production in the SM [7], in modelindependent formalism [8], in models beyond the SM [9], and in supersymmetry (SUSY) [10].

Furthermore, the high luminosity option of the LHC running at $14 \mathrm{TeV}$ (HL-LHC) was approved. It is a legitimate machine to investigate the EWSB sector. In a previous work [11], we showed that even with the high-luminosity LHC one cannot establish the THSC $\lambda_{3 H}$ at the SM value using the most promising decay mode $H H \rightarrow(b \bar{b})(\gamma \gamma)$. Indeed, one can only constrain the THSC to be within $-1.0 \lesssim \lambda_{3 H} \lesssim 7.6$ at 95\% CL [11]. These results were based on a conventional cut-based analysis. In this paper, we show that with the use of BDT method, the significance of the signal can be substantially improved by $80 \%$.

\section{EVENT GENERATION}

The Higgs bosons in the signal event samples are generated on-shell with zero width by POWHEG-BOXV2 $[12,13]$ with the damping factor hdamp set to the default value of 250 to limit the amount of hard radiation. 
TABLE I. Sequence of event preselection criteria applied in this analysis.

\begin{tabular}{ll}
\hline \hline Sequence & \multicolumn{1}{c}{ Event preselection criteria } \\
\hline 1 & Diphoton trigger condition, $\geq 2$ isolated photons with $P_{T}>25 \mathrm{GeV},|\eta|<2.5$ \\
2 & $\geq 2$ isolated photons with $P_{T}>30 \mathrm{GeV},|\eta|<1.37$ or $1.52<|\eta|<2.37, \Delta R_{\gamma \gamma, j \gamma}>0.4$ \\
3 & $\geq 2$ jets identified as b-jets with leading(subleading) $P_{T}>40(30) \mathrm{GeV},|\eta|<2.4, \Delta R_{b b}>0.4$ \\
4 & Events are required to contain $\leq 5$ jets with $P_{T}>30 \mathrm{GeV}$ within $|\eta|<2.5$ \\
5 & No isolated leptons with $P_{T}>25 \mathrm{GeV},|\eta|<2.5$ \\
\hline \hline
\end{tabular}

This code provides NLO distributions matched to a parton shower taking account of the full top-quark mass dependence. The variation of the THSC, $\lambda_{3 H}$, is also allowed in this code. The MadSpin code [14] is used after generating a pair of Higgs bosons in order to decay both Higgs bosons into two bottom quarks and two photons. For parton showering and hadronization, PYTHIA8 [15] is used. Here an appropriate setup provided by POWHEG-BOX-V2 is used to correctly perform a matching of POWHEG-BOX-V2 with PYTHIA8. Finally, fast detector simulation and analysis at the HL-LHC are performed using Delphes3 [16] with the ATLAS template. The parameters in the template are tuned as in Ref. [11]. The signal cross section at NNLO order in QCD is calculated according to $\sigma^{\mathrm{NNLO}}\left(\lambda_{3 H}\right)=K_{\mathrm{SM}}^{\mathrm{NNLO} / \mathrm{NLO}} \sigma^{\mathrm{NLO}}\left(\lambda_{3 H}\right)$, where $\lambda_{3 H}$-dependent NLO cross section of $\sigma^{\mathrm{NLO}}\left(\lambda_{3 H}\right)$ is computed by the use of POWHEG-BOX-V2 and we take $K_{\mathrm{SM}}^{\mathrm{NNLO} / \mathrm{NLO}}=1.116$ [17]. For generation and simulation of backgrounds, we closely follow Ref. [11], except for the use of the post-LHC PDF set of CT14LO [18] and merged cross sections for nonresonant backgrounds. More precisely, for the two main nonresonant backgrounds of $b \bar{b} \gamma \gamma$ and $c \bar{c} \gamma \gamma$, we use the merged cross sections and distributions by MLM matching $[19,20]$ with xqcut and $Q_{\text {cut }}$ set to $20 \mathrm{GeV}$ and $30 \mathrm{GeV}$, respectively. For the remaining nonresonant backgrounds, we are using the cross sections and distributions obtained by applying the generator-level cuts as adopted in Ref. [21] which might provide more reliable and conservative estimation of the nonresonant backgrounds containing light jets [11].

\section{TMVA ANALYSIS}

Before performing a multivariate analysis using TMVA [22] with ROOTV6.18 [23], a sequence of event selections is applied to the signal and background samples, see Table I. We choose these 8 kinematic variables for TMVA:

$$
M_{b b}, \quad P_{T}^{b b}, \quad \Delta R_{b b} ; \quad M_{\gamma \gamma}, \quad P_{T}^{\gamma \gamma}, \quad \Delta R_{\gamma \gamma} ; M_{\gamma \gamma b b}, \quad \Delta R_{\gamma b} .
$$

We observe that significance can be meaningfully improved by judiciously choosing the two photons or two $b$ quarks for the above TMVA variables. For $M_{b b, \gamma \gamma}$ and $P_{T}^{b b, \gamma \gamma}$, in terms of $P_{T}$, we choose the least energetic two photons or two $b$ quarks while the most energetic ones are chosen for $\Delta R_{b b, \gamma \gamma}$ and $M_{\gamma \gamma b b}$. For $\Delta R_{\gamma b}$, on the other hand, we choose the least energetic $b$ and the next-to-the-least energetic photon.

We have scrutinized the normalized distributions of these 8 variables for signal with $\lambda_{3 H}=1$ and backgrounds after applying the pre-selection cuts in Table I. We observe the broad peak around $125 \mathrm{GeV}$ in the $M_{b b}$ distribution of the signal while the peak in the $M_{\gamma \gamma}$ distribution of the signal is very sharp. The signal tends to give larger transverse momenta of $P_{T}^{b b, \gamma \gamma}$ while it populates more in the region of smaller $\Delta R_{b b, \gamma \gamma}$, implying a strong negative correlation between $P_{T}$ and $\Delta R$. Furthermore, the signal has larger $M_{\gamma \gamma b b}$ and its distribution is peaked around $400 \mathrm{GeV}$, and $\Delta R_{\gamma b}$ provides another good discriminant observable.

First, we try various multivariate analysis (MVA) methods provided by TMVA with the default setup for each method. After examining the receiver operating characteristic (ROC) curves for various methods, we find that the BDT-related methods show higher performance with better signal efficiency and stronger background rejection. We choose the best method of BDT for our analysis. Let us first describe our setup for BDT briefly. For each event sample of signal and backgrounds, we randomly divide it into two halves with a default split seed. The first half is used for training and the second one for testing. We use 800 trees and node splitting is allowed only when the number of events in a node is larger than $2.5 \%$ of total number of events of the training sample in order to improve the BDT performance. Maximum tree depth is set to 4 . Training is carried out using adaptive boost with learning rate $\beta=0.5$. One half of the training sample is randomly chosen at the end of each boosting iteration. The cut value on the variable in a node is optimized by comparing the separation index (we choose Gini Index) of the parent node and the sum of the indices of the two daughter ones. Finally, the whole range of the variable is equally gridded into 20 cells.

\section{RESULTS}

In the left panel of Fig. 1, we show the BDT responses obtained using BDT trained for $\lambda_{3 H}=1$ which is to be called $\mathrm{BDT}_{\mathrm{SM}}$ shortly. By validating the BDT distributions for the training sample (dots with error bars) with those for the test sample (histogram), we check that $\mathrm{BDT}_{\mathrm{SM}}$ is not overtrained. In the right panel of Fig. 1, we compare the 
TMVA overtraining check for classifier: BDT

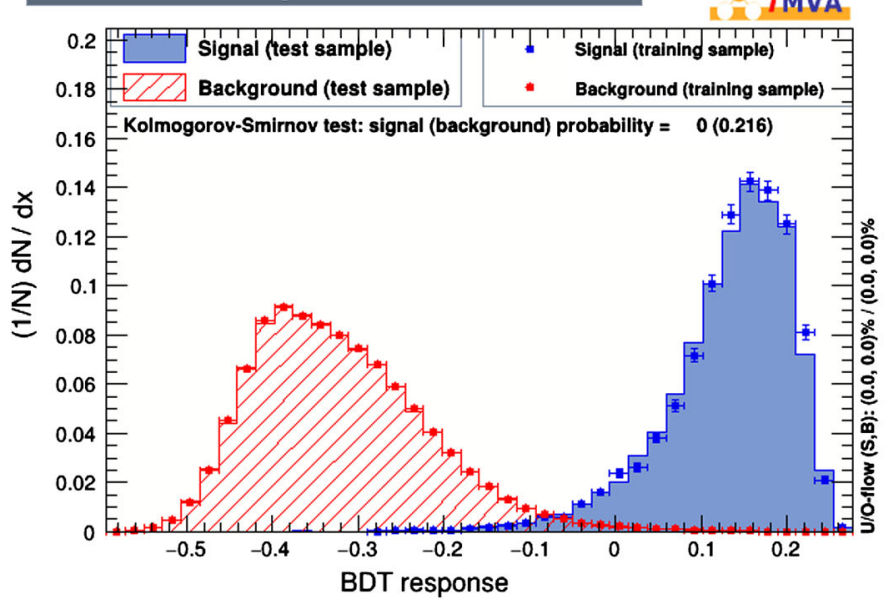

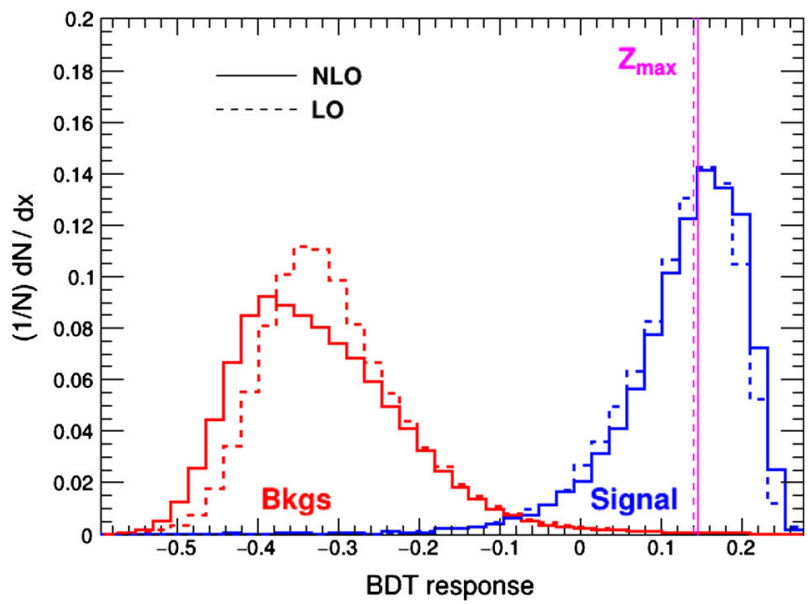

FIG. 1. (Left) Normalized SM BDT responses for test (histogram) and training (dots with error bars) samples. BDT responses for signal (blue) and background (red) samples mostly populates in the regions with positive and negative BDT response, respectively. (Right) Normalized BDT responses for test sample obtained by using NLO (solid) and LO (dashed) distributions of the 8 TMVA input variables. The vertical lines show the position of the optimal cut on the BDT response which maximizes the significance.

BDT responses for the test sample obtained using NLO (solid) and LO (dashed) distributions of the 8 kinematic variables for TMVA. We observe that the NLO BDT distributions provide a bit better separation between signal and background. We denote by vertical lines the positions of the optimal cut on the BDT response which maximizes the significance $Z=\sqrt{2 \cdot[((s+b) \cdot \ln (1+s / b)-s)]}$,

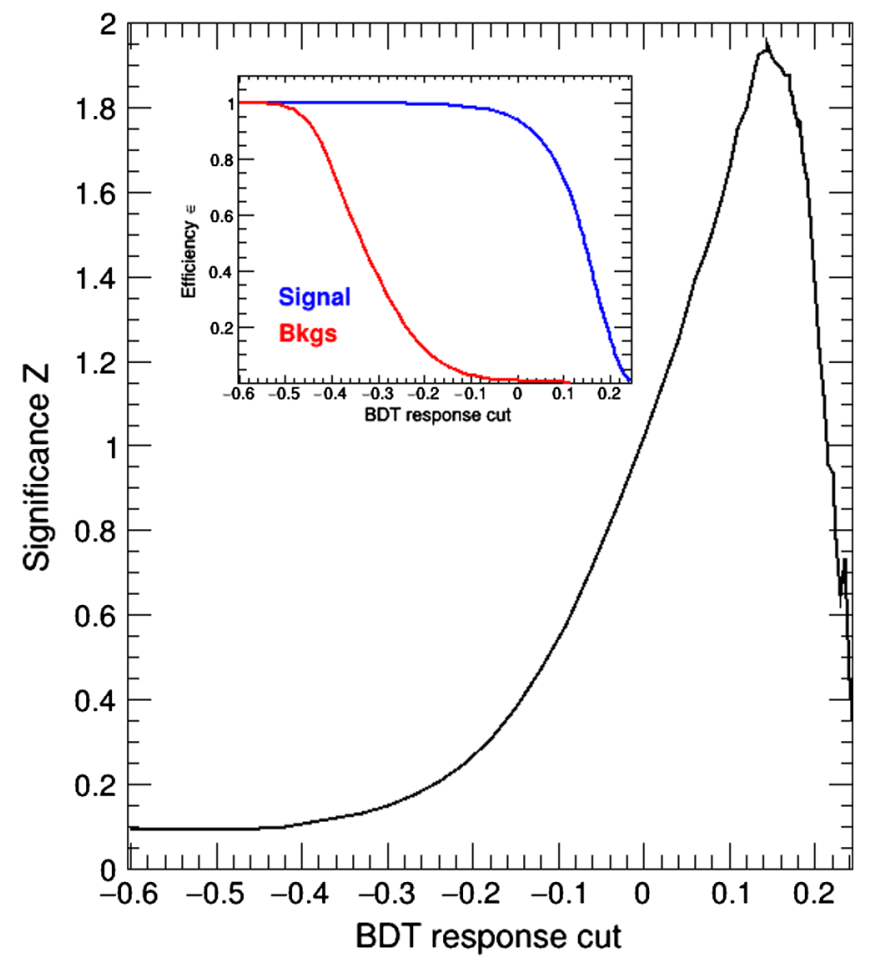

FIG. 2. Signal and background efficiencies (inset) and significance $Z$ as functions of BDT response cut. $\mathrm{BDT}_{\mathrm{SM}}$ is used. where $s$ and $b$ represent the numbers of signal and background events, respectively.

In Fig. 2, using $\mathrm{BDT}_{\mathrm{SM}}$ we show the behavior of signal and background efficiencies and significance $Z$ versus the cut value on BDT response. The significance can reach up

TABLE II. Expected number of signal and background events at the HL-LHC assuming $3000 \mathrm{fb}^{-1}$ using $\mathrm{BDT}_{\mathrm{SM}}$ with the BDT response cut of 0.145 . We consider the four representative values of $\lambda_{3 H}$ for signal and the backgrounds are separated into three categories. For comparisons, we also show the results obtained using the cut-and-count analysis [11].

\begin{tabular}{lrrr}
\hline \hline & \multicolumn{3}{c}{ Expected yields $\left(3000 \mathrm{fb}^{-1}\right)$} \\
\cline { 2 - 4 } & & & Cut-and- \\
Signal and backgrounds & Preselection & $\mathrm{BDT}_{\mathrm{SM}}$ & Count \\
\hline$H(b \bar{b}) H(\gamma \gamma), \lambda_{3 H}=-5$ & 223.22 & 72.73 & 90.19 \\
$H(b \bar{b}) H(\gamma \gamma), \lambda_{3 H}=0$ & 33.69 & 14.63 & 16.70 \\
$\mathbf{H}(\mathbf{b} \overline{\mathbf{b}}) \mathbf{H}(\gamma \gamma), \lambda_{\mathbf{3 H}}=\mathbf{1}$ & $\mathbf{1 7 . 7 7}$ & $\mathbf{8 . 8 5}$ & $\mathbf{9 . 6 3}$ \\
$H(b \bar{b}) H(\gamma \gamma), \lambda_{3 H}=5$ & 26.37 & 4.04 & 6.77 \\
$g g H(\gamma \gamma)$ & 70.72 & 3.94 & 7.04 \\
$t \bar{t} H(\gamma \gamma)$ & 157.20 & 3.64 & 13.14 \\
$Z H(\gamma \gamma)$ & 23.60 & 2.27 & 3.60 \\
$b \bar{b} H(\gamma \gamma)$ & 2.65 & 0.08 & 0.13 \\
$b \bar{b} \gamma \gamma$ & 4676.36 & 3.72 & 10.92 \\
$c \bar{c} \gamma \gamma$ & 3787.00 & 1.20 & 5.41 \\
$j j \gamma \gamma$ & 1015.48 & 0.39 & 2.89 \\
$b \bar{b} j \gamma$ & 10017.91 & 0.82 & 13.91 \\
$c \bar{c} j \gamma$ & 4679.36 & 0.55 & 4.78 \\
$b \bar{b} j j$ & 2517.71 & 0.05 & 3.83 \\
$Z(b \bar{b}) \gamma \gamma$ & 184.07 & 0.32 & 0.88 \\
$t \bar{t}(\geq 1$ leptons) & 7338.84 & 0.35 & 5.09 \\
$t \bar{t} \gamma(\geq 1$ leptons $)$ & 2369.11 & 0.62 & 3.69 \\
Total Background & 36839.99 & 17.94 & 75.31 \\
Significance $Z, \lambda_{3 H}=1$ & & $\mathbf{1 . 9 5}$ & $\mathbf{1 . 0 9}$ \\
\hline \hline & & & \\
\hline \hline & & & \\
& & &
\end{tabular}



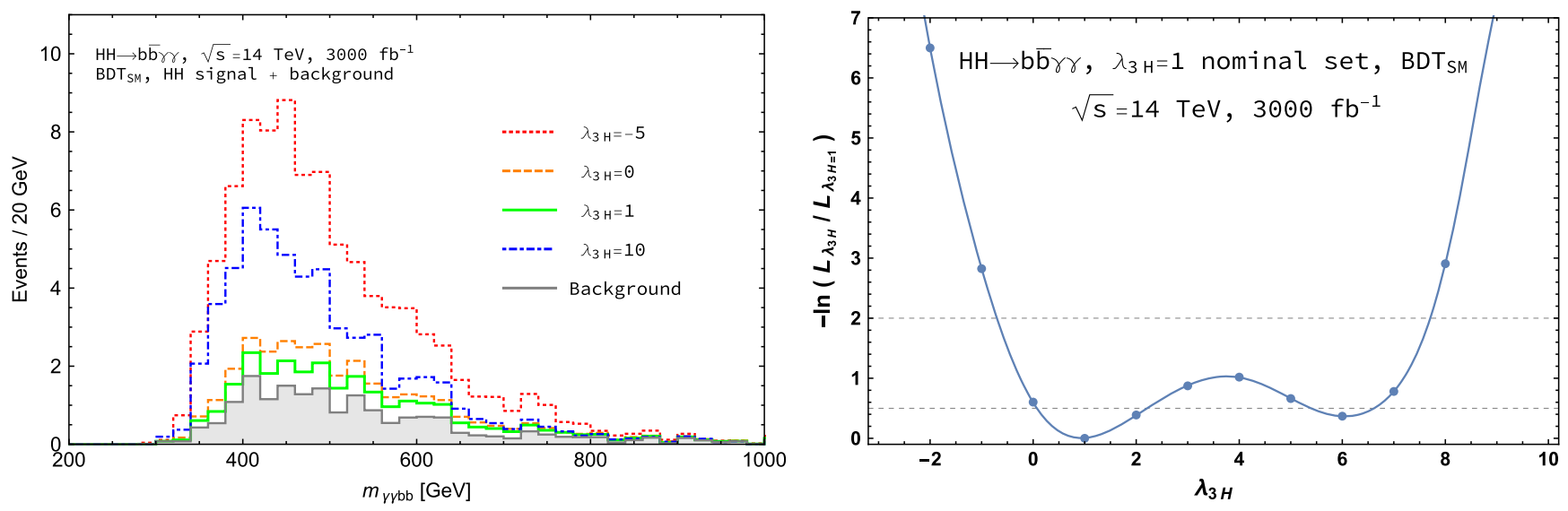

FIG. 3. (Left) $M_{\gamma \gamma b b}$ distributions at HL-LHC for the signal and background combined, assuming $3000 \mathrm{fb}^{-1}$ and using BDT $\mathrm{SM}_{\mathrm{Sith}}$ the BDT response cut of 0.145 . (Right) The relative log likelihood distribution for the nominal value of $\lambda_{3 H}=1$. The dashed line at $0.5(2.0)$ indicate the values corresponding to a $1 \sigma(2 \sigma)$ confidence interval.

to 1.95 when the BDT response is cut at 0.145 , at which, the signal and background efficiencies are 0.50 and $4.9 \times 10^{-4}$, respectively.

In Table II, we present expected number of signal and background events at the HL-LHC assuming $3000 \mathrm{fb}^{-1}$ using $\mathrm{BDT}_{\mathrm{SM}}$ with the BDT response cut of 0.145 . We find 9 signal and 18 background events for $\lambda_{3 H}=1$. Comparing to the results using the cut-and-count analysis [11], the number of signal events decreases by only $10 \%$ while the number of backgrounds by almost $80 \%$, resulting in an increase in significance from 1.09 to 1.95 . Note that the composition of backgrounds changes drastically by the use of BDT. In the cut-and-count analysis, the nonresonant background is about two times larger than the single-Higgs associated background. While, in the BDT analysis, the single-Higgs associated background is larger than the nonresonant one and $t \bar{t}$ associated background becomes negligible. Also, the sensitivity to the THSC can be improved to $1.00<\lambda_{3 H}<6.22$ at $95 \% \mathrm{CL}$, which removes the region of negative $\lambda_{3 H}$ in contrast to the results based on the cut-and-count analysis.

Nevertheless, the significance standing at 1.95 may not be high enough to make a precise measurement of the THSC at the HL-LHC. We implement a likelihood fitting of $M_{\gamma \gamma b b}$ distribution to quantify the uncertainty in the determination of $\lambda_{3 H}$ and to see how much the two-fold ambiguity in the determination could be lifted up. In the left frame of Fig. 3, we show $M_{\gamma \gamma b b}$ distributions for the signal and combined background (shaded histograms) taking four representative values of $\lambda_{3 H}$. In the right frame of Fig. 3, we show the log likelihood distribution obtained by fitting the signal-plus-background $M_{\gamma \gamma b b}$ distributions with the nominal value of $\lambda_{3 H}=1$. The solid line shows the result of a polynomial fitting and we find the $1 \sigma$ CI of $0.1<\lambda_{3 H}<2.2 \cup 5.4<\lambda_{3 H}<6.6$. We observe that the two-fold ambiguity is slightly lifted up by the amount of $\Delta\left[-\ln \left(L_{\lambda_{3 H}} / L_{\lambda_{3 H}=1}\right)\right] \simeq 0.4$.
So far we have used the BDT trained for $\lambda_{3 H}=1$ or $\mathrm{BDT}_{\mathrm{SM}}$ independently of the input value of $\lambda_{3 H}$. Without knowing the value of $\lambda_{3 H}$ a priori, it would be more desirable to use separate BDTs trained for specific values of $\lambda_{3 H}$, which we call $\mathrm{BDT}_{\lambda_{3 H}}$ in short. In Fig. 4, we show the contour plot showing the $1 \sigma$ and $2 \sigma$ CI regions obtained by likelihood fitting of $M_{\gamma \gamma b b}$ distributions. For each value of $\lambda_{3 H}^{\text {in }}$, we use the corresponding $\mathrm{BDT}_{\lambda_{3 H}}$ together with $\lambda_{3 H}=\lambda_{3 H}^{\text {in }}$ nominal set and the BDT response cut is set to

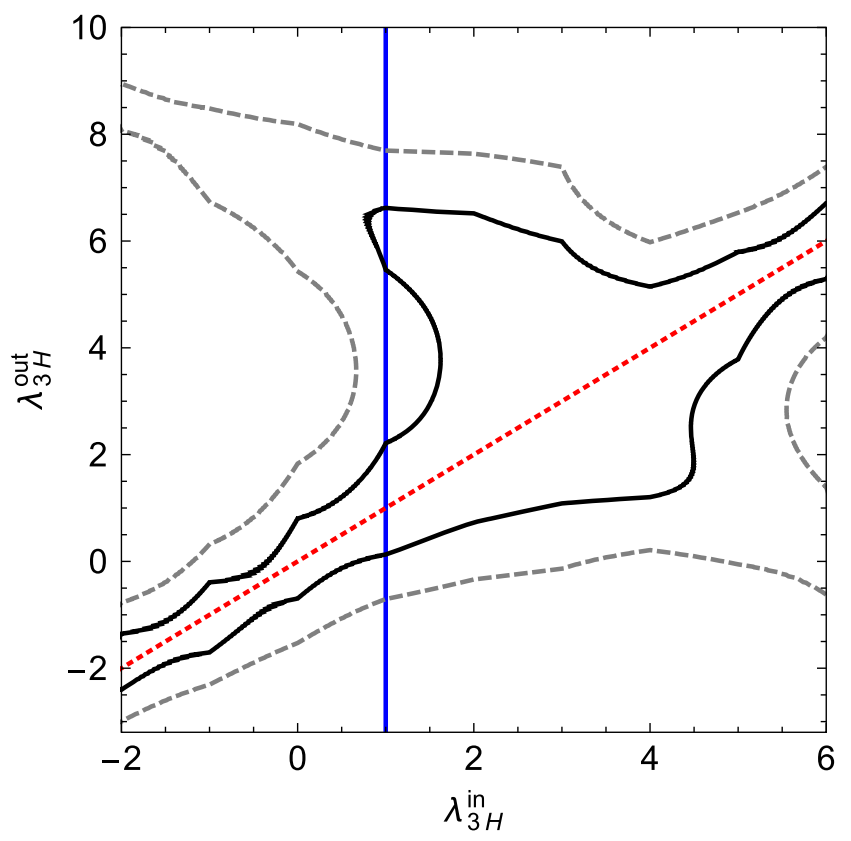

FIG. 4. The 1- and 2- $\sigma$ CI regions versus the input values of $\lambda_{3 H}^{\text {in }}$ at the HL-LHC assuming $3 \mathrm{ab}^{-1}$. The CI regions are obtained by likelihood fitting of $M_{\gamma \gamma b b}$ distributions using $\mathrm{BDT}_{\lambda_{3 H}}$. The solid and dashed lines delimit the 1- and 2- $\sigma$ CI regions, respectively. And the diagonal red dotted line denotes $\lambda_{3 H}^{\text {out }}=\lambda_{3 H}^{\text {in }}$ and the vertical blue line at $\lambda_{3 H}^{\text {in }}=1$ indicates the case shown in the lower frame of Fig. 3 with $\mathrm{BDT}_{\mathrm{SM}}$. 
TABLE III. NLO and LO results obtained using BDT $\mathrm{BM}_{\mathrm{SM}}$ and comparison with the recent ATLAS result [24]. $\mathrm{Z}_{\max },\left.s\right|_{Z_{\max }}$, and $\left.b\right|_{Z_{\max }}$ denote the significance, the number of signal events, and the number of background events, respectively, obtained after applying the BDT cut which maximizes the significance. Also compared are the $95 \% \mathrm{CL}$ and $1 \sigma \mathrm{CI}$ ranges of $\lambda_{3 H}$.

\begin{tabular}{lccc}
\hline \hline & \multicolumn{2}{c}{ BDT $_{\mathrm{SM}}$} & ATLAS_2018 [24] \\
\cline { 2 - 4 } & $\mathrm{MLM} \oplus \mathrm{NLO}_{\text {dist }}$ & MLM $\oplus \mathrm{LO}_{\text {dist }}$ & Ref. [21] $\oplus \mathrm{LO}_{\text {dist }}$ \\
\hline $\mathrm{Z}_{\max }$ & 1.95 & 1.79 & 2.1 \\
$\left.s\right|_{\mathrm{Z}_{\max }}$ & 8.85 & 8.61 & 6.46 \\
$\left.b\right|_{Z_{\max }}$ & 17.94 & 20.44 & 6.8 \\
$95 \% \mathrm{CL}$ & $(1.00,6.22)$ & $(0.87,6.55)$ & $(1.3,6.2)$ \\
$1 \sigma \mathrm{CI}$ & $(0.1,2.2) \cup(5.4,6.6)$ & $(0.1,2.3) \cup(5.3,6.7)$ & $(-0.1,2.4)$ \\
\hline \hline
\end{tabular}

maximize significance. Using $\mathrm{BDT}_{\lambda_{3 H}}$, the $95 \% \mathrm{CL}$ region is narrowed into $1.01<\lambda_{3 H}<5.42$ at $95 \% \mathrm{CL}$. Compared to that obtained using $\mathrm{BDT}_{\mathrm{SM}}$, we observe the noticeable changes of $Z$ for $\lambda_{3 H} \gtrsim 4$. We also find there exists a bulk region of $0.5 \lesssim \lambda_{3 H} \lesssim 4.5$ in which it is hard to pin down the THSC, see the $1-\sigma$ error region delimited by solid lines in Fig. 4.

In Fig. 1, we show that using the NLO distributions of signal for TMVA may lead to better results. For quantitative comparisons we use the LO distributions for TMVA and find that the significance can reach up to 1.79 with about 9 signal and 20 background events. The details of the NLO and $\mathrm{LO}$ results based on $\mathrm{BDT}_{\mathrm{SM}}$ are presented in Table III where we also make comparison with the recent ATLAS result without systematic uncertainties [24] in which the LO distributions of signal and the generator-level cuts adopted in Ref. [21] are used for TMVA. ${ }^{1}$

\footnotetext{
${ }^{1}$ A similar approach using BDTs was explored in Ref. [25] previously. But they consider only a part of the background processes considered in this work and, being different from the case of ATLAS, it is difficult to make a direct comparison of our results with theirs.
}

\section{CONCLUSIONS}

Higgs-pair production is the most useful avenue to probe the EWSB sector. We have studied in great detail, with the help of machine learning, the sensitivity of measuring the THSC $\lambda_{3 H}$ that one can expect at the HLLHC with $3000 \mathrm{fb}^{-1}$. With TMVA one can improve upon the signal-to-background significance over the traditional cut-based analysis. In this work, we have shown that the significance is improved by about $80 \%$ and found a narrower range of $\lambda_{3 H}$ below the sensitivity. With BDTs trained for each value of $\lambda_{3 H}$, we found the bulk region further down to $0.5 \lesssim \lambda_{3 H} \lesssim 4.5$ in which one cannot pin down the THSC. For the Supplemental Material, we refer to Ref. [26].

\section{ACKNOWLEDGMENTS}

We thank Chih-Ting Lu for initial participation. This work was supported by the National Research Foundation of Korea Grant No. NRF-2016R1E1A1A01943297 (J. C., J. S. L., J. P.), No. NRF-2018R1D1A1B07051126 (J. P.), and by the MoST of Taiwan under Grant No. 1072112-M-007-029-MY3 (K. C.).
[1] G. Aad et al. (ATLAS Collaboration), Phys. Lett. B 716, 1 (2012); S. Chatrchyan et al. (CMS Collaboration), Phys. Lett. B 716, 30 (2012).

[2] K. Cheung, J. S. Lee, and P. Y. Tseng, J. High Energy Phys. 05 (2013) 134; Phys. Rev. D 90, 095009 (2014).

[3] K. Cheung, J. S. Lee, and P. Y. Tseng, J. High Energy Phys. 09 (2019) 098.

[4] E. W. N. Glover and J. J. van der Bij, Nucl. Phys. B309, 282 (1988); D. A. Dicus, C. Kao, and S. S. D. Willenbrock, Phys. Lett. B 203, 457 (1988); T. Plehn, M. Spira, and P. M. Zerwas, Nucl. Phys. B479, 46 (1996); 531, 655(E) (1998); A. Djouadi, W. Kilian, M. Muhlleitner, and P. M. Zerwas, Eur. Phys. J. C 10, 45 (1999); S. Dawson, S. Dittmaier, and M. Spira, Phys. Rev. D 58, 115012 (1998); U. Baur,
T. Plehn, and D. L. Rainwater, Phys. Rev. D 67, 033003 (2003); T. Binoth, S. Karg, N. Kauer, and R. Ruckl, Phys. Rev. D 74, 113008 (2006).

[5] U. Baur, T. Plehn, and D. L. Rainwater, Phys. Rev. D 68, 033001 (2003); Phys. Rev. Lett. 89, 151801 (2002); Phys. Rev. D 69, 053004 (2004).

[6] J. Baglio, A. Djouadi, R. Grber, M. M. Mhlleitner, J. Quevillon, and M. Spira, J. High Energy Phys. 04 (2013) 151; J. Grigo, J. Hoff, K. Melnikov, and M. Steinhauser, Nucl. Phys. B875, 1 (2013); V. Barger, L. L. Everett, C. B. Jackson, and G. Shaughnessy, Phys. Lett. B 728, 433 (2014); W. Yao, arXiv:1308.6302.

[7] C. Englert, F. Krauss, M. Spannowsky, and J. Thompson, Phys. Lett. B 743, 93 (2015); T. Liu and H. Zhang, 
arXiv:1410.1855; D. E. Ferreira de Lima, A. Papaefstathiou, and M. Spannowsky, J. High Energy Phys. 08 (2014) 030; V. Barger, L. L. Everett, C. B. Jackson, and G. Shaughnessy, Phys. Lett. B 728, 433 (2014); E. Asakawa, D. Harada, S. Kanemura, Y. Okada, and K. Tsumura, Phys. Rev. D 82, 115002 (2010); A. Papaefstathiou, L. L. Yang, and J. Zurita, Phys. Rev. D 87, 011301 (2013); A. Papaefstathiou, Phys. Rev. D 91, 113016 (2015); R. Frederix, S. Frixione, V. Hirschi, F. Maltoni, O. Mattelaer, P. Torrielli, E. Vryonidou, and M. Zaro, Phys. Lett. B 732, 142 (2014).

[8] K. Nishiwaki, S. Niyogi, and A. Shivaji, J. High Energy Phys. 04 (2014) 011; M. Gouzevitch, A. Oliveira, J. Rojo, R. Rosenfeld, G. P. Salam, and V. Sanz, J. High Energy Phys. 07 (2013) 148; M. J. Dolan, C. Englert, and M. Spannowsky, J. High Energy Phys. 10 (2012) 112; A. Azatov, R. Contino, G. Panico, and M. Son, Phys. Rev. D 92, 035001 (2015); N. Liu, S. Hu, B. Yang, and J. Han, J. High Energy Phys. 01 (2015) 008; F. Goertz, A. Papaefstathiou, L. L. Yang, and J. Zurita, J. High Energy Phys. 04 (2015) 167; R. Grober, M. Muhlleitner, M. Spira, and J. Streicher, J. High Energy Phys. 09 (2015) 092; F. Goertz, A. Papaefstathiou, L. L. Yang, and J. Zurita, J. High Energy Phys. 06 (2013) 016; R. Contino, M. Ghezzi, M. Moretti, G. Panico, F. Piccinini, and A. Wulzer, J. High Energy Phys. 08 (2012) 154; C. R. Chen and I. Low, Phys. Rev. D 90, 013018 (2014); R. S. Gupta, H. Rzehak, and J. D. Wells, Phys. Rev. D 88, 055024 (2013); D. Goncalves, T. Han, F. Kling, T. Plehn, and M. Takeuchi, Phys. Rev. D 97, 113004 (2018); Q. H. Cao, B. Yan, D. M. Zhang, and H. Zhang, Phys. Lett. B 752, 285 (2016); Q. H. Cao, G. Li, B. Yan, D. M. Zhang, and H. Zhang, Phys. Rev. D 96, 095031 (2017); H. J. He, J. Ren, and W. Yao, Phys. Rev. D 93, 015003 (2016); C. T. Lu, J. Chang, K. Cheung, and J. S. Lee, J. High Energy Phys. 08 (2015) 133.

[9] S. Dawson, E. Furlan, and I. Lewis, Phys. Rev. D 87, 014007 (2013); M. Gillioz, R. Grober, C. Grojean, M. Muhlleitner, and E. Salvioni, J. High Energy Phys. 10 (2012) 004; V. Barger, L. L. Everett, C. B. Jackson, A. Peterson, and G. Shaughnessy, Phys. Rev. Lett. 114, 011801 (2015); M. J. Dolan, C. Englert, and M. Spannowsky, Phys. Rev. D 87, 055002 (2013); G. D. Kribs and A. Martin, Phys. Rev. D 86, 095023 (2012); A. Arhrib, R. Benbrik, C. H. Chen, R. Guedes, and R. Santos, J. High Energy Phys. 08 (2009) 035; C. O. Dib, R. Rosenfeld, and A. Zerwekh, J. High Energy Phys. 05 (2006) 074; R. Grober and M. Muhlleitner, J. High Energy Phys. 06 (2011) 020; J. M. No and M. Ramsey-Musolf, Phys. Rev. D 89, 095031 (2014); B. Hespel, D. Lopez-Val, and E. Vryonidou, J. High Energy Phys. 09 (2014) 124; S. M. Etesami and M. M. Najafabadi, Phys. Rev. D 92, 073013 (2015); T. Corbett,
A. Joglekar, H. L. Li, and J. H. Yu, J. High Energy Phys. 05 (2018) 061.

[10] C. Han, X. Ji, L. Wu, P. Wu, and J. M. Yang, J. High Energy Phys. 04 (2014) 003; U. Ellwanger, J. High Energy Phys. 08 (2013) 077; J. Cao, Z. Heng, L. Shang, P. Wan, and J. M. Yang, J. High Energy Phys. 04 (2013) 134; B. Bhattacherjee and A. Choudhury, Phys. Rev. D 91, 073015 (2015); D. T. Nhung, M. Muhlleitner, J. Streicher, and K. Walz, J. High Energy Phys. 11 (2013) 181.

[11] J. Chang, K. Cheung, J. S. Lee, C. T. Lu, and J. Park, Phys. Rev. D 100, 096001 (2019).

[12] G. Heinrich, S. P. Jones, M. Kerner, G. Luisoni, and E. Vryonidou, J. High Energy Phys. 08 (2017) 088.

[13] G. Heinrich, S. P. Jones, M. Kerner, G. Luisoni, and L. Scyboz, J. High Energy Phys. 06 (2019) 066.

[14] P. Artoisenet, R. Frederix, O. Mattelaer, and R. Rietkerk, J. High Energy Phys. 03 (2013) 015.

[15] T. Sjstrand, S. Ask, J. R. Christiansen, R. Corke, N. Desai, P. Ilten, S. Mrenna, S. Prestel, C. O. Rasmussen, and P.Z. Skands, Comput. Phys. Commun. 191, 159 (2015).

[16] J. de Favereau, C. Delaere, P. Demin, A. Giammanco, V. Lemaître, A. Mertens, and M. Selvaggi (DELPHES 3 Collaboration), J. High Energy Phys. 02 (2014) 057.

[17] M. Grazzini, G. Heinrich, S. Jones, S. Kallweit, M. Kerner, J. M. Lindert, and J. Mazzitelli, J. High Energy Phys. 05 (2018) 059.

[18] S. Dulat, T.-J. Hou, J. Gao, M. Guzzi, J. Huston, P. Nadolsky, J. Pumplin, C. Schmidt, D. Stump, and C.-P. Yuan, Phys. Rev. D 93, 033006 (2016).

[19] M. L. Mangano, M. Moretti, F. Piccinini, and M. Treccani, J. High Energy Phys. 01 (2007) 013.

[20] J. Alwall et al., Eur. Phys. J. C 53, 473 (2008).

[21] ATLAS Collaboration, Report No. ATL-PHYS-PUB-2017001, 2017, http://cds.cern.ch/record/2243387.

[22] A. Hoecker et al., Proc. Sci., ACAT2007 (2007) 040 [arXiv: physics/0703039].

[23] R. Brun and F. Rademakers, Nucl. Instrum. Methods Phys. Res., Sect. A 389, 81 (1997); See also http://root.cern.ch/.

[24] ATLAS Collaboration, Report No. ATL-PHYS-PUB-2018053.

[25] A. Alves, T. Ghosh, and K. Sinha, Phys. Rev. D 96, 035022 (2017).

[26] See Supplemental Material at http://link.aps.org/ supplemental/10.1103/PhysRevD.101.016004 for a summary of information on the signal and background processes, the normalized distributions of the eight kinematic variables for the SM signal and backgrounds, and the receiver operating characteristic (ROC) curves for various MVA methods. 